\title{
Protein folding dynamics study for protein-protein interactions
}

\author{
Patil A.G. ${ }^{1}$, Ojha M.D. ${ }^{1}$, Bhandari P.A. ${ }^{2}$, Kulkarni S. ${ }^{3}$ \\ ${ }^{1}$ V.E.S., College of Arts, Science and Commerce, Chembur, Mumbai-400 071, India \\ ${ }^{2}$ P.T.V.A's Sathaye College, Vile Parle (E), Mumbai-400 057, India \\ ${ }^{3}$ Padmashree Dr. D.Y. Patil University, Navi Mumbai, 400614, India
}

\begin{abstract}
Protein folding is useful and challenging problem in structural biology. Molecular dynamics (MD) and simulation has proved to be a paramount tool and was widely used to study protein structures, folding kinetics and thermodynamics and structure-stability-function relationship. It was also used to help engineering and designing new proteins, and to answer even more general questions for target validation, such as the evolution principle of protein families. MD simulation is still undergoing rapid developments. The first trend is to toward developing new coarse-grained models and studying larger and more complex molecular systems such as protein-protein complex and their assembling process, and structure; the second trend is toward building high resolution protein models and explore more detailed and accurate pictures of protein folding and the associated processes.
\end{abstract}

Keywords- Protein folding, Molecular dynamics, Protein Quality Control, protein-protein interactions

\section{Introduction}

The study of basic protein science provides the insight of role of proteins in health and diseases and relates the study of protein to the context of modern high throughput data streams of genomics and proteomics [7]. A protein molecule is an ultra micro machine [11], which forms the building block and functions as catalyst to keep up the biochemical activity, specific binding to promoters leads to constitutive or inducible expression of genes, act as structural elements of complex system in association with other biomolecules, signal transmission and regulates unfolding programs of development. The structure of protein is intimately linked to their function and hence predicting and studying the basic protein structure and function using bioinformatics, databases and computational method has paved the way to design novel proteins with specific desired character.

\section{Advances of protein molecular biology and cell biology}

Recent advances in protein structural biology, coupled with new developments in human genetics, have opened the door to understanding the molecular basis of many metabolic, physiological, and developmental processes in human biology. Medical pathologies, and their chemical therapies, are increasingly being described at the molecular level. For single-gene diseases, and some multi-gene conditions, identification of highly correlated genes immediately leads to identification of covalent structures of the actual chemical agents of the disease, namely the protein gene products. Once the primary sequence of a protein is ascertained, structural biologists work to determine its threedimensional, biologically active structure, or to predict its probable fold and/or function by comparison to the database of known protein structures [1]. The researches in the genetic field regarding the functioning of proteins in a biomolecule system have helped in determining whether it affects the metabolism of the system. Such as in Alzheimer's disease the beta amyloid protein aggregation leads to neurodegeneration and hence understanding the processing, trafficking and turnover of beta amyloid protein will pave the way for better drug discovery for treatment of Alzheimer's disease [2]. In recent years, the detailed study of some of the virulent antigens causing dreaded infections and pandemic breakouts such as SARS and SWINE FLU caused by H1N1 virus has lead to the search of preventive strategies and cure of such infections. These studies now offer potential avenues for developing more efficient vaccine strategies. These include identification of viral antigens and viral receptors, viral genome sequencing and development of cDNA clone using recombinant technology [3]. The advent of protein science in molecular and cellular level has not only benefited the arena of human health and diagnostics but also played a pivotal role in agricultural field. The study on various regulatory proteins that help to induce the gene expression under certain environmental conditions and thus leading to growth of plant, have been proved to be immensely useful in crop development, secondary metabolite production and resistance from pathogens to plants. Regulatory proteins that are under the control of plant hormones auxins and cytokinins and other environmental factor play an important role in overall plant development and crop yield. The detailed knowledge of such proteins and the use of transformation techniques has lead to potential application in agriculture [4,5]. The advent of protein engineering made it possible to redesign an enzyme to tackle tasks such as capability to synthesis only a specific antibiotic or to activate only a particular antibody in case of particular immune disorder. For this, recent researches have been done to analyze accurate 3 dimensional structure of the subject protein from 
X-ray crystallography and reactivity of substrates. From this information, the shortcomings of native enzyme and improvements required can be identified down to detail on the amino acid substitution to be made and then modifying the respective protein at molecular level [6].

\section{Protein folding dynamics}

Understanding the process according to which a linear polypeptide chain acquires its highly structured, biological functional native fold is one of the most fundamental problems in science. The considerable insight into the physics of the folding process was gained from the study of simplified protein models like the Framework model [46, 47,48]. Hydrophobic collapse model [49]. Nucleation model [50, 51]. Under the principle of two state transitions i.e. form unfolded to folded state, it stated that protein folding is spontaneous, reversible under ideal conditions, non random and highly co-operative. However, these models were still far from the native structure and cannot be studied in detail due to millisecond resolution of most techniques [8] [1214]. The classical view of protein folding is based on the thermodynamics [Anfinsen's] [9] v/s kinetics [Levinthal] [10]. Debate considers protein a unique system and requires detailed atomic description of its folding pathway. The new view reconciles through a unified theory that considers folding to take place in a multidimensional energy landscape. It focuses on essential milestone of folding namely unfolded, transitions and intermediate states as dynamic ensembles of conformation corresponding to local minima and saddle points of energy landscape [8]. Energy landscape and folding funnel model Rather than describing folding processes the Folding Funnel describes overall shape of the free energy landscape and its ruggedness relates to protein folding kinetics. It gives the Energy pattern of all possible conformation of protein and Correlation between the conformational space and relative free energies of the protein. It suggests, there is no one specific pathway that a protein folds along. Instead, a protein runs down a multidimensional funnel towards the final structure. There are a large number of possible intermediates between the fully unfolded and fully folded protein [16]. Folding occurs through the progressive organization of ensembles of structures [shown here for the src-SH3 domain (left)] on a funnel-shaped free energy landscape (right). Conformational entropy loss during folding is compensated by the free energy gained as more native interactions are formed. Kinetics is determined by the local roughness of the landscape, relative to thermal energy. Key interactions in early folding (dashed circle) coincide, for this protein, with experimentally determined regions. The computational model [in silico] of protein folding has proved to be a helping hand. It involves the application of lowlevel representation computer models to the study of folding process in which each protein residue is represented by one or more particles. Computer programs have been made use of to simulate protein folding and generate precise 3-D structure of any protein from its amino acid sequence by using protein database. Some of the recent models studied using computational methods are

- Charge centre model.

- Lattice model.

- Rosetta model.

- de novo model.

- Stochastic model.

Features:

- Gives an account of energetics and protein folding process.

- Applied in prediction of protein structure.

- Simple representation.

- It can sample the conformational space efficiently.

- Less expensive than other methods.

- Simulate relatively large proteins over a small period, (nanoseconds) [52].

This has marked a decisive step towards understanding biological complexcity, hierarchical organization, self-assembly and regulation in biological system. It has impact in human health and welfare in designing new proteins and drugs, through understanding of structure and function of hundred's of protein sequence that are being discovered everyday. The design of new drug is important in treatment of amyloid diseases, caused by misfolded proteins that form amyloid fibrils. In Creutzfeldt-Jacob disease, an infection caused prion proteins. Prions are proteins that are found in two forms, one that folds normally and another that aggregates into amyloid like deposits in the brain. Recently there has been an approach to avoid protein misfolding by designing a protein that binds specifically to intermediate that make amyloid, preventing from aggregating. Alzheimer's, Parkinson's disease, sickle cell anemia and cancer are the diseases caused by protein misfolding [15]. In addition, the physical aspects of protein dynamics have been studied using large number of experiments and theoretical methods to get a reliable physical picture using Myoglobin as a model protein. The comparison of experiments at unphysiologically low temperatures with those at physiological temperatures were done to understand the protein dynamics in different conditions which are essential for the biological function as they are activated only above a characteristic temperature, which is often called the dynamical transition temperature. The studies were done using Mössbauer absorption spectroscopy and incoherent neutron scattering and it was shown that at low temperatures the molecules are 
trapped in 'rigid' states and with increasing temperatures, the probability increases to reach states that allow more flexibility [18]. Now, Researchers in the School of Pharmacy are studying the structure and dynamics by unfolding proteins using nanotechnology devices to pull them apart and simulations of these experiments allows them to view the protein at the atomistic level as it unfolds. In collaboration with researchers at Cornell University, the School has developed a method to simulate protein unfolding over vastly extended timescales, and using a new concept of "milestoning", the project will follow the motion of the protein for periods several hundred times longer than has been achieved to date. The high performance computer allows, for the first time, the simulation of the whole unfolding process. The simulations provide a view into how nature selected the amino acids and their sequence to engineer the shape, dynamics and function of the protein, and opens new avenues of research into evolution and opportunities for advances in treatment of diseases, such as Alzheimer's and diabetes [19]. Protein folding was studied using techniques like circular dichroism spectroscopy, dual polarisation interferometry, vibrational circular dichroism combined with $\mathrm{X}$-ray diffraction and fourier transform instrument [FFT]. Modern studies use the new 4B8 beamline provides UV-VUV light in the wavelength range from 360 to $120 \mathrm{~nm}$, which enables two kinds of spectroscopy measurements: synchrotron radiation circular dichroism spectroscopy and VUV excited fluorescence spectroscopy used in protein secondary structure studies. It uses a laserinduced temperature jump for protein folding dynamics using circular dichroism as a probe [53].

\section{Protein stability \\ Protein stability helps to study the connection between a protein structure and the forces that maintain it. The stability of protein structure is described by the difference in free energy between the unfolded and native states. The primary forces involved in protein stability are VanderWaal's interaction, hydrogen bonding, electrostatic interaction, configurational entropy. Water plays a crucial role in protein stability causing the hydrophobic or the solvation effect[54, 55]. The stability of proteins is measured using Differential Scanning Colorimeter [DSC] [22].}

\section{Stability of proteins used as biotherapeutics}

A stable, correctly folded protein is an absolute requirement for a successful biotherapuetic. Proteins are increasingly used as therapeutic agent, hence the knowledge of their thermal and conformational stability are important for the successful development of these proteins.
Recently antibodies and Fc-conjugate proteins have been studied using DSC for their potential use as therapeutics [24]. In anti cancer drug design such as human carbanic anhydrases and human chaperon protein $\mathrm{Hsp90}$ that helps in correct folding of proteins are analysed at various conditions of temperature and pressure to study their stability [23]. It has been shown that stereoelectronic effects stabilize folded proteins and using this approach simple derivatives of collagen are synthesized which is more stable than natural collagen [25-29]. Substitutions of solvent-exposed amino acids on the surfaces of proteins are seen to have little if any effect on protein stability or structure, leading to the view that it is the rigid parts of proteins that are critical for folding and stability. In recent years, there is substantial progress in developing methods to engineer proteins of enhanced stability. One such studies show that the accurate prediction of protein stability changes resulting from single amino acid mutations is important for understanding protein structures and designing new proteins. This studies is done using support vector machines to predict protein stability changes for single amino acid mutations leveraging both sequence and structural information and the web server for predictions of protein stability changes upon mutations (MUpro), software, and datasets are used [31].

\section{Protein Quality Control}

In living cells, both newly made and preexisting polypeptide chains are at constant risk for misfolding and aggregation. In accordance with the wide diversity of misfolded forms, elaborate quality-control strategies have evolved to counter these inevitable mishaps. Recent reports describe the removal of aggregates from the cytosol; reveal mechanisms for protein quality control in the endoplasmic reticulum; and provide new insight into two classes of molecular chaperones, the Hsp70 system and the AAA+ (Hsp100) unfoldases. Proteins recognized as non-native in the $E R$ will be removed and degraded by a process termed ERAD. ERAD of aberrant proteins is accompanied by various changes of cellular organelles and results in protein folding diseases thus synthetic chaperones can be employed to rescue diseasecausing trafficking defects of aberrant proteins [21].

\section{Heart failure and protein quality control}

Development of congestive heart failure is due to inadequate regulation of cardiac protein quality control. Cardiomyocytes are continuously challenged to efficiently and properly fold nascent polypeptides, traffic them to their appropriate cellular locations, and keep them from denaturing in the face of normal and pathological stimuli, as misfolded or unfolded proteins can be disastrous 
cardiomyocytes, have developed a multilayered protein quality control system carried out primarily by chaperones, and ubiquitin-protease system mediated proteolysis maintain proper protein conformation, reorganize and remove misfolded or aggregated polypeptides [34-35].

\section{Role in muscle development}

Myofibrillogenesis involves ubiquitin-dependent degradation of regulatory muscle proteins. The chaperone family of UCS proteins (UNC-45CRO1-She4p) is required for the proper function of myosin motors. Mutations in the myosindirected chaperone unc-45, a founding member of this family, lead to disorganization of striated muscle by disrupting the assembly of sarcomeric proteins such as desmin and actin [36].

\section{Treatment of neurodegenerative disorders}

As studied in the Parkinson's disease, the mitochondrial proteins are regulated by the ubiquitin-dependent protein quality control and where proteasome function is defective, accumulation of mitochondrial proteins may cause a collapse of the mitochondrial network. Interference with protein quality control by genetic or chemical manipulation in mitochondria and endoplasmic reticulum often results in aggregate formation and neurodegeneration. Hence, the proper regulation of quality control helps to overcome neurodegenerative disorders and its study may provide a target for therapeutic intervention [37, 38].

\section{Protein quality control during aging}

The BAG3 / BAG1 [Bcl-2 associated anthanogen act as modulators which regulate the macroautophagic and proteosomal pathway, respectively for degradation of polyubiquitinated proteins] ratio is elevated in neurons during aging and thus BAG3 is important in mediating recruitment of macroautophagy pathway to maintain protein homeostasis in protein quality control system to avoid aggregation prone condition characteristic of aging [30].

\section{Protein - Protein interaction}

Protein protein interactions are intrinsic to virtually every cellular process such as DNA replication, transcription, translation, splicing, secretion, cell cycle control. Therefore, it is important to identify proteins that interact and assess the strengths of these interactions. The protein protein interaction in metabolic enzymes like in glycosylation pathway and during DNA replication is associated with large structure called protein machines. Transient protein protein inetraction is seen in protein modification such as protein kinase, protein phosphatse, acyl transferase and proteases with their substrate protein in fundamental processes like cell growth and cell cycle; also, in recruitment and assembly of transcription complex, to specific promoters, the transport of proteins across membranes, the folding of native proteins catalyzed by chaperonins [56].

\section{Kinetic properties}

This can be reflected in altered binding of substrates, altered catalysis, altered allosteric properties of the complex.[57]. Thus, the interaction of proliferating-cell nuclear antigen with DNA polymerase alters processivity of the polymerase [58]. The interaction of succinate thiokinase and a-ketoglutarate dehydrogenase lowers the $\mathrm{Km}$ for succinyl coenzyme $\mathrm{A}$ by 30 fold [59].

\section{Substrate channeling}

The paradigm for this type of complex is tryptophan synthetase from Neurospora crassa. It is a complex of two subunits, each of which carries out one of the two steps of reaction (formation of indole from indole 3-glycerol phosphate,followed by conversion of indole to tryptophan). The intermediate indole is noncovalently bound, but it is preferentially channeled to form tryptophan [60].

\section{Formation of a new binding site}

Thus, an ADP site forms at the interface of the a and $b$ subunits of Escherichia coli F1-ATPase [61]. Yeast hexokinase binds one ATP molecule at the interface of the asymmetric homodimer [62] and phosphofructokinase from Bacillus stearothermophilus binds both fructose 6phosphate and ADP at the interface between subunits [63].

\section{Inactivate a protein}

The interaction of phage P22 repressor with its antirepressor [64]; the interaction of trypsin with trypsin inhibitor[65]., and the interaction of phage T7 gene 1.2 protein with $E$. coli dGTP triphosphohydrolase [66].

\section{Specificity of a protein for its substrate}

Interaction of lactalbumin with lactose synthase lowers the $\mathrm{Km}$ for glucose by 1,000-fold, and the interaction of transcription factor with RNA polymerase direct the polymerase to different promoters [67].

\section{Protein Interaction Databases}

The Protein-Protein Interaction Server is a tool for analysing the protein-protein interface between any two polypeptide chains in the three dimensional structure of a protein complex. An organism specific database is The Drosophila Protein Interaction Map (PIM) database. The Biomolecular Interaction Network Database is designed to store full descriptions of interactions, molecular complexes and pathways. The 
Database of Interacting Proteins holds data on functional interactions between yeast proteins that have been inferred from a selection of computational approaches [68].

\section{Protein Transduction}

Protein transduction is described as internalisation of proteins into the cell, from the external environment. This process relies on the inherent property of a small number of proteins and peptides(10-16 residues long) of being able to penetrate the cell membrane. It is an emerging technology with potential applications in gene therapy for delivery of therapeutic proteins into target cells [39, 40]. The three most commonly used protein transduction vehicles are the antennapedia peptide, the herpes simplex virus VP22 protein and HIV TAT protein transduction domain. Linking the protein domains covalently to compounds, peptides, antisense peptide, nucleic acids able them enter any cell type in a receptorand transporter-independent fashion. These fusions are delivered to all tissues, even crossing the blood-brain barrier thus important to address the issues regarding treatment of human disease [33].

\section{Protein transduction used for sex determination}

Transduced proteins are folded properly and can mediate their respective functions in their hosts. Thus this approach is used to study the molecular events of gonadogenesis in mice using two reporter proteins, TAT- -gal and -gal fusion proteins with and without the TAT protein transduction domain (PTD) respectively. The transduced protein was distributed evenly in females and heterogeneously in male gonads after seminiferous tubule differentiation in which the amount of reporter protein was higher outside than inside the tubules. Hence such protein delivery technique is useful in studies designed to evaluate the sex determining or as new tool to study of cellular ageing and senescence [42, 44].

\section{Treatment of diabetes}

This technology is useful for the treatment of diabetes. In islet isolation and transplantation, cell-permeable peptides deliver anti-apoptotic molecules to protect islets. Another peptide provides immunosuppression for fully mismatched islet allografts, thus suggesting that peptide drugs could lead to outcome improvement for pancreatic islet transplantation. In addition, this technology facilitates the differentiation of stem cells into insulin-producing cells [43].

\section{Elimination of tumor cells}

The production and functional delivery of the Cassava prodrug system eliminate tumor cells both in vitro and in vivo [45]. Thus generic protein transduction systems have the potential to provide affordable, safe and efficient medicines in the not-too-distant future.

\section{Conclusion}

The major goal of basic protein studies is the generation of novel molecules since the revolution introduced by genetic engineering in protein science. The second wave of innovation can be considered the protein engineering technology as it produces tailor-made proteins redesigned such as to make it suitable for desired work. The analysis of function and properties of basic protein like protein interaction, protein quality control, protein stability and use of new methods like protein tranduction using various physical, chemical and computational methods has proved to be a helping hand. The researches carried out in above processes can be further applied in various fields of biotechnology like human health and diagnostics, industries and agriculture.

References

[1] Woodward C. (1997) J. Advances in Molecular and Cell Biology 22A, 1-2.

[2] Sambamurti K., Greig N.H., Lahiri D.K.(2002) J. Neuromolecular medicine.,1-31.

[3] Caren J. and Atreya C.D.(2005) J. Virology.,2-35.

[4] Eugene, Nester.W., DeshPal S. (1993) J.Advances in molecular genetics of plant microbe interaction.,3-14.

[5] Suckling.C.J. (1990) J. Enzyme chemistry: Impact and application.,172-178.

[6] Petsko G.A., Ringe D. (1989) J. Physical Chemistry.,4217.

[7] Patrica F., Faisca N. (2000) J .Soft Condensed Matter: New Research.,195217.

[8] Anfinsen C.B. (1973) J. Science.,81:223 230.

[9] Levinthal C.J. (1968) J.Chemistry and Physics.,65:44-45.

[10] Tanford Reynolds C. (2001) J. Natures.,3.

[11] Pauling Corey L. (1951) J. Natl. Acad. Sci.,37:729- 740.

[12] Minton Curr P. (2000) J. Structural Biology.,34-39.

[13] Harlt F. U.(1996) J. Nature.,381:571 -581.

[14] Prusiner S.B. Proc.(1998) J. Natl. Acad. Sci., 245-272.

[15] Dinner Sali A., Smith A., Dobson L.J., Karplus C.M. (2000) J.TIBS., 331-339.

[16] Radford S. E. (2000) J.Trends in Biochemical Sciences., 525-539.

[17] Maisuradze G.G., Liwo A., Scheraga H.A. (2003) J.Physical aspects of protein dynamics., 89-123.

[18] Kachalo S., Lu H.M., Liang J. (2006) J. Phys. Rev. lett.,96. 
[19] Royer C. (2004) Protein protein interaction., 1-31.

[20] Jürgen R., Gary H., Jingyu F., Kiyoko H. (2007) J. Histochemical Cell Biology.,123.

[21] Jie Wen, Yijia Jiang, and Linda N. (2008) American Pharmaceutical Review., 4549.

[22] Winum J.Y., Scozzafava A., Montero J.L., Supuran C.T. (2007) Curr Top Med Chem. 7(9):835-48.

[23] Fredric Girard, Vincent Vachon, Gabrielle Pr fontaine, Lucie Marceau, Jean-Louis Schwartz, Luke Masson and Raynald Laprade (2009) Applied and Environmental Microbiology, 75(2), 359365.

[24] Choudhary A., Gandla D., Krow G. R., Raines R. T. (2009) J. Am. Chem. Soc., $131,7244$.

[25] Shoulders M. D., Kamer K. J., Raines R. T. (2009) J. Bioorg. Med. Chem. Lett.,19.

[26] Gottlieb D., Morin S. A., Jin S., Raines R. T. (2008) J. Mat. Chem., 18, 3865.

[27] Kotch F. W., Guzei I. A., Raines R. T. (2008) J. Am. Chem. Soc., 130:2952.

[28] Shoulders M. D., Guzei I. A., Raines R. T. (2008) J. Biopolymers ., $89: 443$.

[29] Martin G., Parvana H., Murat K., Uwe W., Ulrich H. and Christian B. (2009) J. EMBO., 899-901.

[30] Matthews B.W. (1993) J. Annual Review of Biochemistry, 62, 139-160.

[31] Krishnamurthy R. (1999) J. BioPharm., 31:34-38.

[32] Cheng J., Randall A., Baldi P. (2006) Proteins, 62(4):1125-32.

[33] Xuejun W., Jeffrey R. (2006) J. American Heart Association, 99:1315.

[34] Sudha K. (2006) J. American Heart Association, 100: 1276.

[35] Johnny K., Thomas L. and Thorsten H. (2008) J. Trends in Cell Biology, 264272.

[36] Scheper W., Hoozemans J. (2009) J.Current medical chemistry, 615-26.

[37] Susanne R., Harish C., Patrick S., Gregor M., Rejko K., Jörg B. S. and Doris G. (2008) J. Molecular Physiology of the Brain.,12681-12685.

[38] Ford K. G., Souberbielle B. E., Darling D. and Farzaneh F. (2001) J. Gene Therapy, 1-14.

[39] Schwarze S.R., Hruska K.A., Dowdy S.F. (2000) J. Trends in Cell Biology, 290-5.

[40] Li Y., Yue L., Taketo T., Lau Y.-F.C. (2003) J. Cytogenet Genome Res., 101:237241.

[41] Ford K. G., Darling D., Souberbielle B., Farzaneh F. (2000) J. Mechanism of ageing and development., 121: 1-31: 113-121.
[42] Hirofumi N. and Shinich (2006) J. HepatoBilliary pancreatic surgery, 13, 306-13.

[43] Nils L., Jens M. Kelm, René R., David G., Valentin D., Jean B. W. and Martin Fu. (2006) J. Nucleic acid Res., 34.

[44] Wurm F.M.(2004) J. Nat. Biotechnol.,,22:1393 -1398.

[45] Ptitsyn B.(1973) J.Doklady Akademii Nauk SSSR., 210, 1213-1215.

[46] Kim P.S. and Baldwin R. S. (1990) J. Annu. Rev. Biochem.,59, 631-660.

[47] Dyson H. J. and Wright P. E. (1993) J. Curr. Opin. Struc. Biol.,3:1:60-65.

[48] Dill K. A. ,Bromberg S., Yue K. Z., Fiebig K., Yee D. P. Chan. (1995) J Protein Sci., 4: 561-602.

[49] Abkevich I., Gutin A. M. and Shakhnovich E. I. (1994) J.Biochemistry., 33:1002610036 .

[50] Wetlaufer D. B. (1973) J. Natl Acad. Sci. USA., 697-701.

[51] Brenner S. E. and Berry A. (1994) Protein Sci., 3(10): 1871-1882.

[52] Bork P., Downing A.K., Kieffer B., Campbell I.D. (1996) Q Rev Biophys., 29(2):11967.

[53] Shirley B. (1995) J. Protein stability and folding., 51-52.

[54] Eric M. P. And Stanley F. (1995) J.American Society for Microbiology., 94-123.

[55] Monod, Wyman (1965) J. Mol. Biol., 12:88118.

[56] Porpaczy Z., Sumegi B. and Alkonyi I. (1983) J.Biochim.Biophys.Acta.,749:172-179.

[57] Prelich G., Tan C. K., Kostura M., Mathews M. B. (1989) J.Nature, 326:517-520.

[58] Yanofsky C. and Rochmeler M. (1958) J. Biochim. Biophys. Acta., 28:640-645.

[59] Weber J., Lee F., Wilke-Mounts S., Grell E. and Senior A. E. (1993) J. Biol. Chem. 268:6241-6247.

[60] Steitz T.A., Anderson W.F., Fletterick R.J. and Anderson C.M. (1977) J. Biol. Chem. 252:4494-4500.

[61] Evans P. R., Farrants G.W. and Hudson P. J. (1981) J. Philos. Trans. R. Soc.,5362.

[62] Vincent J. P. and Lazdunski M.(1972) J. Biochemistry.,11:2967-2977.

[63] Susskind M. M. and Youderian P. (1987) J. Gene Development.,204-212.

[64] Vinson C. R., Hai T. and Boyd S. M. (1993) J. Genes Dev., 1047-1058.

[65] Hill R. L., and Brew K. (1975) J. Adv. Enzymol., 43:411-490.

[66] Wixon J. (2001) J. Comparative and Functional Genomics., 2: 338-343. 